\title{
Evolution et mobilité des sédiments superficiels de l'estran occidental de la baie du Mont Saint-Michel
}

\author{
Chantal Bonnot-Courtois*, Philippe Bassoullet**, Pierre Le Hir**, \\ Bernadette Tessier***, Florence Cayocca**, Agnès Baltzer*** \\ * Chargée de recherche CNRS, UMR CNRS 8586 PRODIG, Laboratoire de \\ Géomorphologie et Environnement littoral. 15, boulevard de la mer, \\ 35800 Dinard. chantal.bonnot@ephe.sorbonne.fr \\ ** Ingénieurs de recherche, IFREMER, DYNECO/PHYSED. BP 70. \\ 29280 Plouzané.pbassoul@ifremer.fr ; plehir@ifremer.fr ; fcayocca@ifremer.fr. \\ *** Chargée de recherche CNRS et Maître de conférences, UMR CNRS 6143 \\ M2C "Morphodynamique Continentale et Côtière", Université de Caen, \\ 14000 Caen.bernadette.tessier@unicaen.fr ; agnes.baltzer@unicaen.fr
}

Résumé

La baie du Mont Saint-Michel se caractérise dans sa partie occidentale par de vastes replats de marée (tidal flats) vaseux à sableux, soumis à l'action dominante et quotidienne des courants tidaux ainsi qu'à l'action, plus épisodique, des houles. Afin de mieux comprendre et définir les rythmes et l'ampleur des processus "érosion - sédimentation" qui s'opèrent sur de tels estrans, un suivi du niveau du sédiment a été réalisé à trois échelles de temps, le long de trois radiales transversales, longues de plusieurs kilomètres et représentatives des différents environnements sédimentaires de cette partie occidentale de la baie. Les relevés mensuels, effectués en vive-eau, montrent que l'amplitude générale des remaniements est relativement faible (généralement inférieure à $5 \mathrm{~cm}$ ) et variable d'Ouest en Est : importants à Cancale et à Vildé- Hirel, ils sont beaucoup plus faibles à Cherrueix. A certaines périodes de l'année, en particulier hivernales, l'ensemble de l'estran connaît des phases d'érosion de plusieurs centimètres, rapidement suivies de phases de sédimentation de même amplitude. Un équilibre spatial semble s'établir entre les deux radiales les plus occidentales, une phase d'érosion des vases superficielles à Hirel coïncidant avec une phase de sédimentation à Cancale.

\begin{abstract}
The western part of the Mont Saint-Michel Bay is characterized by very extended mud- to sand-flats, predominantly subject to tidal currents, episodically to wave action. In order to define and quantify the rhythm and amplitude of the processes of erosion and sedimentation on such broad tidal flats, measurements of sediment elevation have been performed along three cross-shore profiles. Each profile, few kilometers long, is representative of the different sedimentary environments of the western bay. Monthly measurements, realized during high spring tides, evidence that the amplitude of reworking is generally low $(< \pm 5 \mathrm{~cm})$, and variable from west to east : relatively high at Cancale and Vildé-Hirel, it is much lower at Cherrueix. During storm events, especially during winter season, the whole flat experiences severe erosion of decimetric scale, which is however
\end{abstract}


rapidly followed by accretion of similar amplitude. A spatial equilibrium is also observed between the two westernmost profiles : when mud erosion occurs at Vildé-Hirel, accretion takes place at Cancale.

Mots Clefs: Régime mégatidal, Estran sablo-vaseux, Bilan érosion-sédimentation, Key words: Tidal Sandy-mud flat, Erosion-sedimentation budget,

\section{Introduction}

Le chantier «Capacité trophique de la Baie du Mont Saint-Michel» du Programme National Environnement Côtier a pour objet d'une part d'identifier les probabilités d'échanges entre les espèces benthiques dans la baie proprement dite et dans les systèmes tourbillonnaires du Golfe normand-breton, et d'autre part de déterminer la capacité trophique de la zone conchylicole de la partie occidentale de la baie. Le thème hydro-sédimentaire de ce chantier avait pour objectif de caractériser les transports dissous et particulaires et de mettre en place une modélisation numérique devant constituer la base physique de la modélisation écologique.

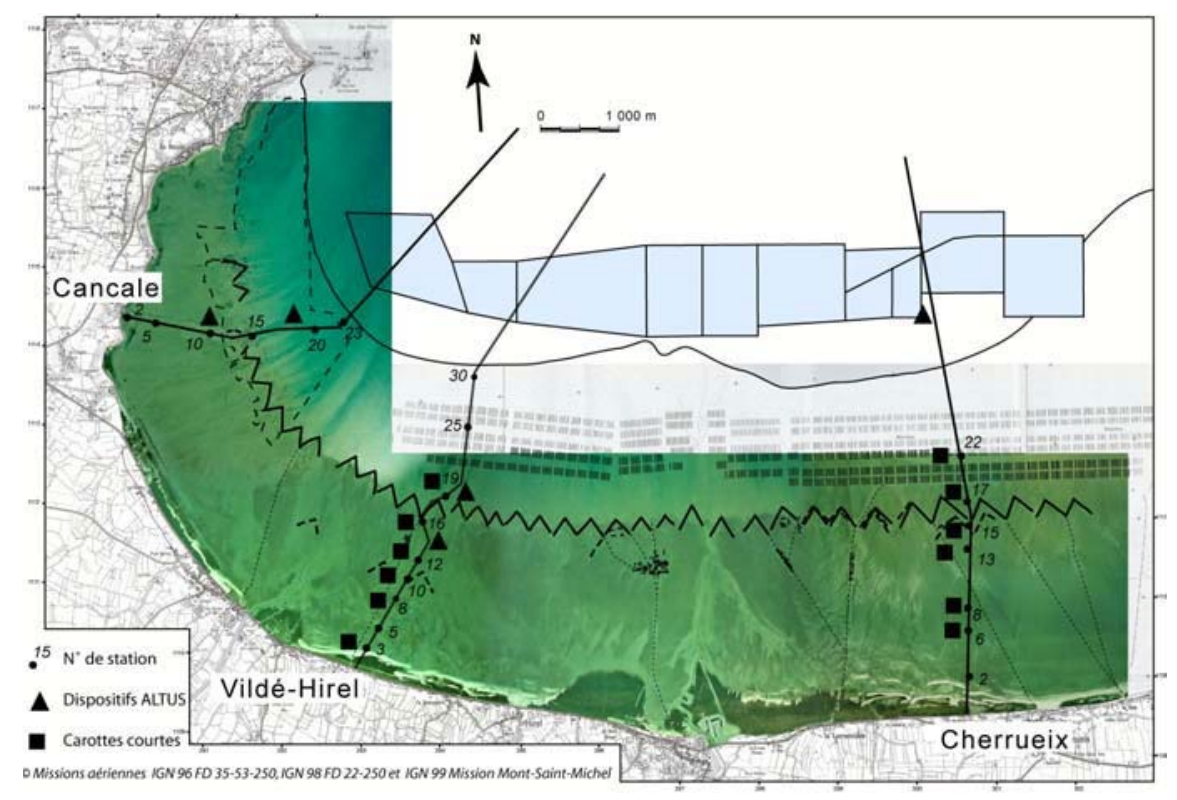

Figure 1 : Morphologie de l'estran occidental de la baie du Mont Saint-Michel et implantation des dispositifs du suivi le long des trois radiales.

La partie occidentale de la baie du Mont Saint-Michel est caractérisée par un très large estran vaseux à sableux (figure 1), évoluant en régime mégatidal sous l'action permanente des courants de marée ([1], [2]). Ces courants de type alternatifs dans la partie centrale de la baie, s'atténuent et prennent un caractère giratoire dans la baie de Cancale. De même, les houles dominantes de NW sont très largement amorties à l'Ouest alors que leur action est significative au centre de la baie. Elle y induit la construction de bancs sablo-coquilliers de grande taille dans la partie haute de l'estran ([3]), et contribue au remaniement général de la surface du tidal flat. 
Afin de mieux caractériser les rythmes et l'ampleur des processus érosion sédimentation qui s'opèrent sur de tels replats de marée, un suivi du niveau du sédiment a été réalisé dans cette partie occidentale de la baie, à plusieurs échelles de temps.

\section{$\underline{\text { 2. Méthodologie }}$}

Pour les évolutions sédimentaires à court terme (cycle tidal journalier), des dispositifs ALTUS (figure 2a) de mesures acoustiques en continu des variations du niveau du sédiment ont été implantés en certaines stations (figure 1) sur plusieurs mois ([4]). Ces mesures permettent de suivre les rythmes d'érosionsédimentation et de les mettre en relation avec les cycles tidaux et/ou les épisodes de plus forte agitation.

Pour le suivi à moyen terme (cycle lunaire), des dispositifs de type jalons permettant de mesurer le niveau du sédiment (figure $2 \mathrm{~b}$ ) ont été implantés en des stations espacées de $150 \mathrm{~m}$ le long de trois radiales transversales, longues de 3 à $4 \mathrm{~km}$, représentatives des différents environnements sédimentaires de l'estran occidental (figure 1). Les relevés ont été effectués en vive-eau, à un rythme mensuel au cours des années 2002 et 2003, et trimestriel à partir de 2004. La précision des mesures est en moyenne de $\pm 3 \mathrm{~mm}$ et varie en fonction de la nature sableuse ou vaseuse du sédiment et du ressuyage de la surface de l'estran.
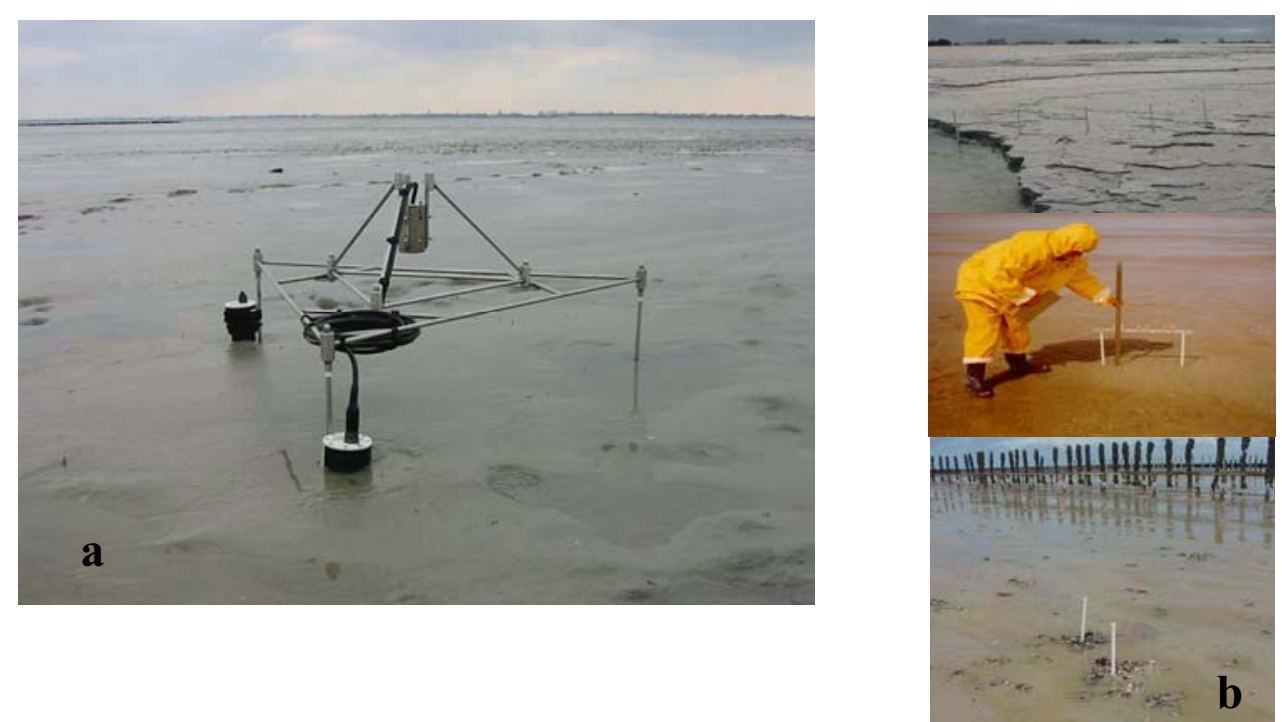

Figure 2 : Dispositif ALTUS (a) et stations d'implantation de jalons (b) pour le suivi moyen terme du niveau du sédiment

Pour les évolutions à long terme (échelle pluri-annuelle) des carottes courtes $(20 \mathrm{~cm})$ ont été prélevées en certaines stations le long des radiales (figure 1) afin de caractériser les faciès sédimentaires associés aux processus de remaniement. 


\section{Cadre morpho-sédimentaire}

Les trois radiales suivies correspondent à différents environnements sédimentaires ([5], [6]), représentés respectivement d'Ouest en Est par :

un mud flat en baie de Cancale, constitué essentiellement de vases fines peu consolidées (figure 3). La partie moyenne et basse de l'estran est occupée par des installations ostréicoles. La pente générale de l'estran est de 2,5\% ;

$>$ un tidal flat sablo-vaseux dans le secteur de Vildé-Hirel (figure 3) où des vasières occupent la partie médiane de l'estran et passent progressivement à des faciès plus sableux dans la partie basse de l'estran, occupée par ailleurs par des anciennes pêcheries et par des installations mytilicoles. La pente du moyen estran est de $3,1 \%$, celle du bas estran, plus faible, de $2 \%$;

un sand flat au centre de la baie à Cherrueix, dominé par des faciès de sables fins tout le long de la radiale (figure 3). Les pêcheries et les lignes de bouchots occupent l'ensemble du bas estran. La pente générale de l'estran est de 3,4 \%o.
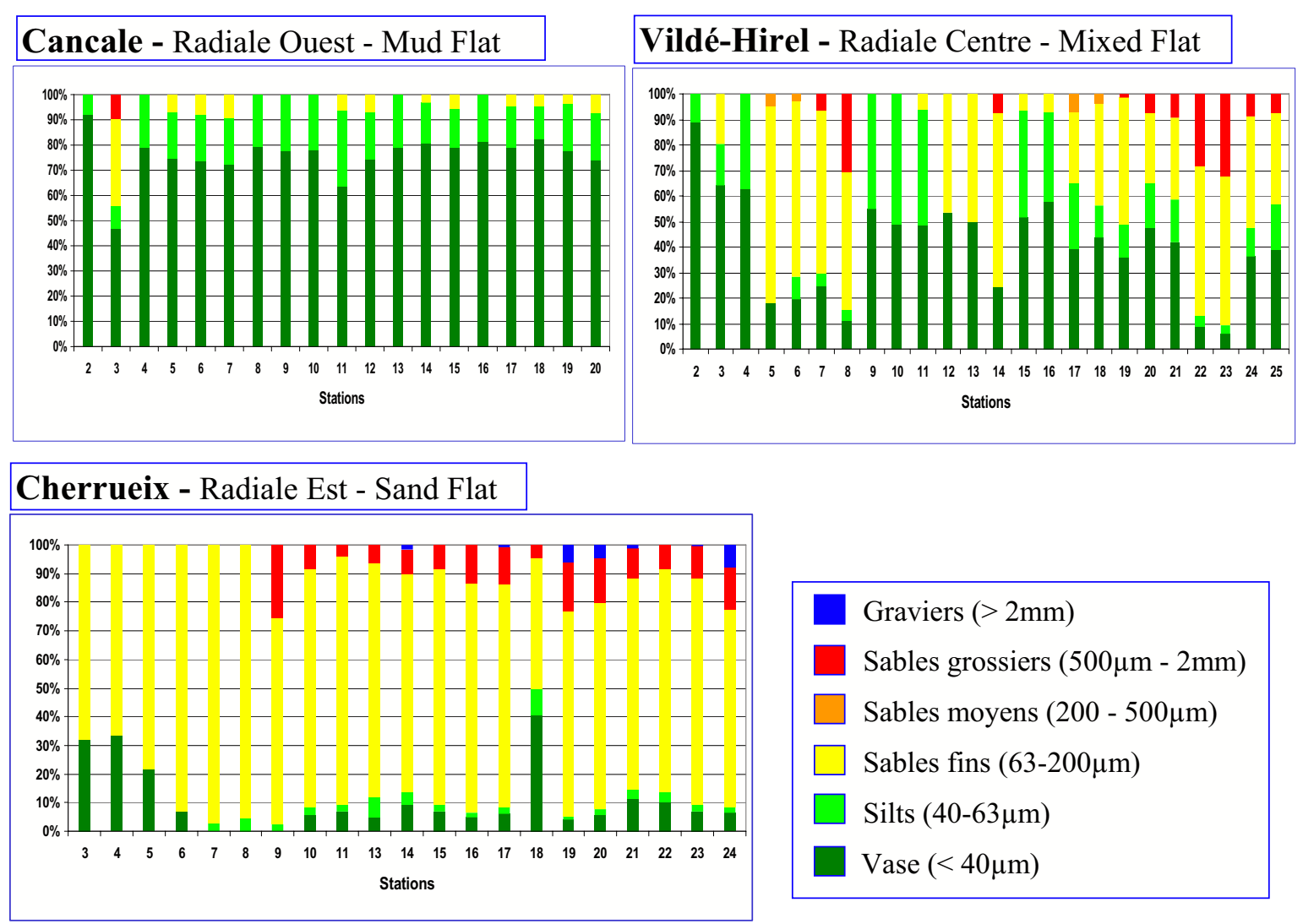

Figure 3 : Evolution granulométrique du sédiment superficiel du haut estran (Stations 2-3) vers le bas estran (Stations 20-25), le long des trois radiales 


\section{Résultats}

\subsection{Les évolutions sédimentaires à court terme}

L'exploitation des enregistrements ALTUS a permis de suivre l'évolution du sédiment superficiel et de détecter une éventuelle "couche crème" temporaire. La cadence d'acquisition, l'enregistrement simultané de la hauteur d'eau et les corrélations avec les conditions météorologiques (vitesse et direction des vents) permettent la mise en évidence des périodes pendant lesquelles se produisent les remaniements ou les dépôts. Une quantification est ainsi possible selon la nature du forçage en jeu, la précision des mesures étant de l'ordre de $\pm 2 \mathrm{~mm}$.
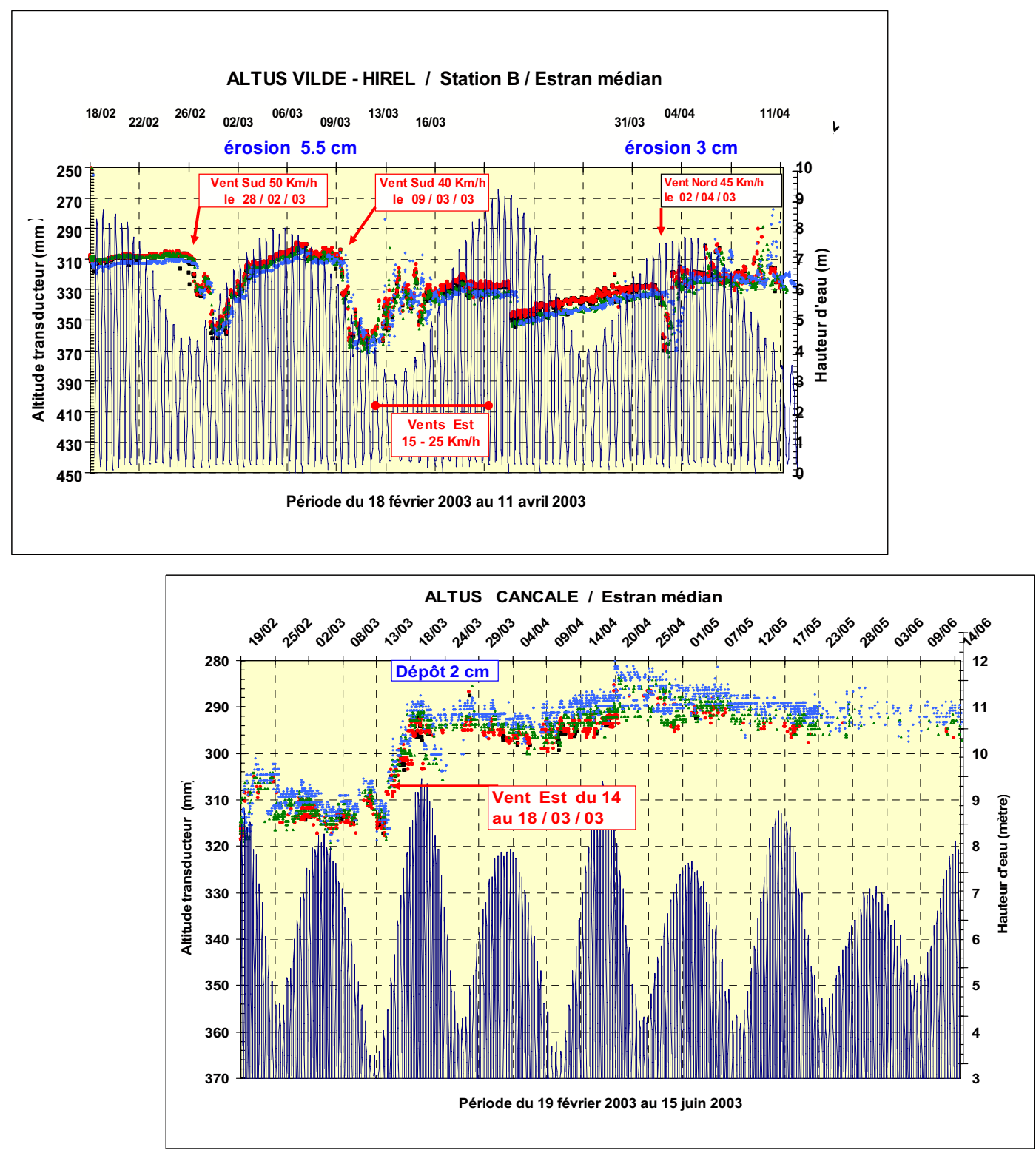

Figure 4 : Suivis ALTUS en amont des installations ostréicoles et des pêcheries au niveau de l'estran médian de Vildé-Hirel et de Cancale

[les différentes teintes du niveau sédimentaire correspondent à diverses interfaces caractérisant des degrés de compaction du sédiment] 
Pour la radiale Vildé-Hirel, les variations notables du niveau de sédiment concernent le secteur situé en amont des pêcheries (figure 4). L'enregistrement de l'altimètre relève en 2 jours des érosions de $5,5 \mathrm{~cm}$ lors d'épisodes de vents de secteur Sud. Ce phénomène est renforcé s'il est conjugué à des marées de fortes amplitudes. Les vents de secteur Est fournissent, durant une semaine, de fortes charges en suspension. La recharge de l'estran est continue fin mars - début avril seulement perturbée par un coup de vent le 02 avril 2003, décapant $3 \mathrm{~cm}$.

L'enregistrement altimétrique sur l'estran médian de la radiale Cancale (figure 4) permet de quantifier $(2 \mathrm{~cm})$ le dépôt d'une couche de vase sous l'action de vents d'Est durant 6 jours (mi-mars 2003). Cet enregistrement permet également d'écarter l'action tidale dans ce transit sédimentaire.

\subsection{Les évolutions à moyen terme : le bilan "érosion-sédimentation » le long des trois radiales}

Les résultats du suivi depuis 2002 , synthétisés sur la figure 5, montrent que :

$>$ l'amplitude des variations de niveau du sédiment est généralement faible $(<5 \mathrm{~cm})$. Localement, des variations de plus forte amplitude sont mesurées $(10 \mathrm{~cm})$;

$>$ les remaniements sont de l'ordre de $\pm 5 \mathrm{~cm}$ à Cancale et à Vildé-Hirel, mais inférieurs à $\pm 2 \mathrm{~cm}$ à Cherrueix.

$>$ une phase d'érosion des vases superficielles à Vildé-Hirel correspond à une phase de sédimentation à Cancale (entre novembre et décembre 2002). Conformément aux observations de terrain, ceci semble indiquer un équilibre spatial entre les deux radiales les plus occidentales;

$>$ le bilan érosion - sédimentation est à l'équilibre au cours du temps :

- à l'échelle saisonnière : en période hivernale, des phases d'érosion de plusieurs cm se produisent sur l'ensemble de l'estran mais sont rapidement suivies par des phases de sédimentation de même amplitude (entre mars et septembre 2004 à Cancale).

- à l'échelle pluri-annuelle, sur toute la période du suivi (3 ans).

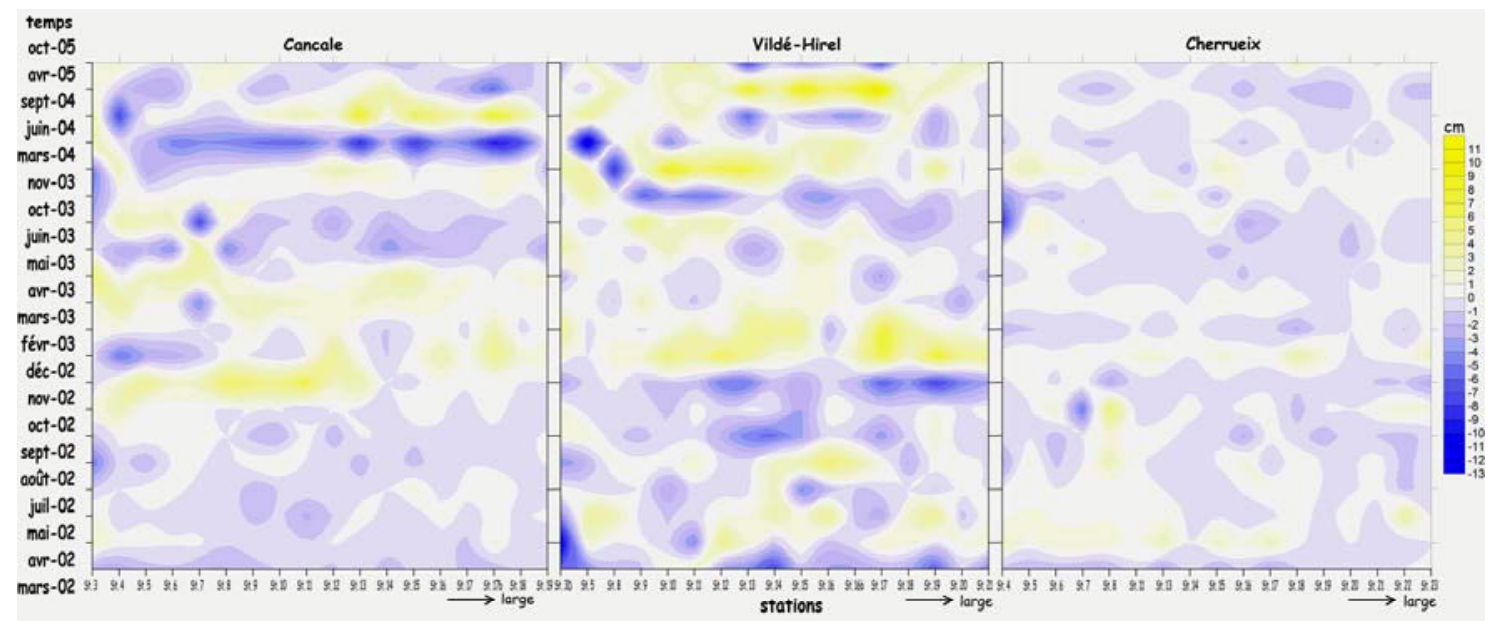

Figure 5 : Remaniements sédimentaires le long des trois radiales entre 2002 et 2005. Echelle des couleurs : Bleu - érosion, Blanc - stabilité, Jaune - sédimentation [en abscisse : succession des stations du haut vers le bas estran, en ordonnée : date des relevés] 


\subsection{Les évolutions à long terme : enregistrements sédimentaires des processus}

Seules les radiales de Vildé-Hirel et de Cherrueix ont fait l'objet de prélèvement de carottes courtes pour l'analyse des successions sédimentaires qui montrent (figure 6) :

- une assez faible variabilité générale de faciès, en accord avec la répartition des sédiments superficiels, représentés par des sables coquilliers, des sables fins à moyens et des vases ([7]);

- la préservation des drapages de vases est meilleure à Vildé-Hirel qu'à Cherrueix, soulignant que l'énergie est globalement plus faible vers l'Ouest ;

- à Vildé-Hirel, la préservation des drapages est meilleure dans la partie moyenne et basse de l'estran que dans la partie haute soumise aux remaniements par les houles.

- quelle que soit la radiale considérée, y compris celle de Cancale, il n 'y a pas de relation directe entre l'amplitude des remaniements et la cote altimétrique de l'estran.

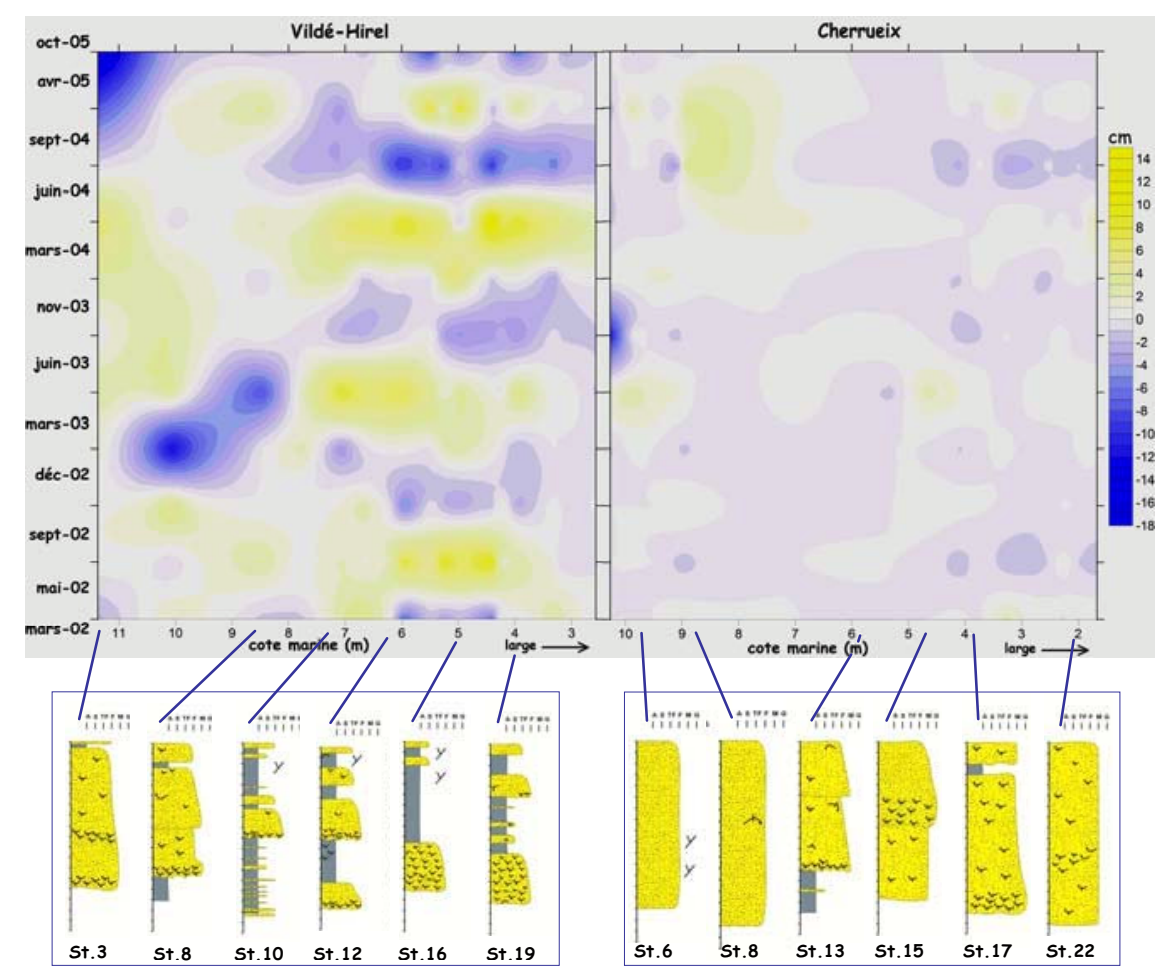

Figure 6 : Relation entre les enregistrements sédimentaires par carottages courts et les remaniements le long des radiales de Vildé-Hirel et Cherrueix.

\section{Conclusion}

Le suivi des processus "érosion-sédimentation" réalisé à plusieurs échelles de temps a permis de détailler la dynamique des sédiments superficiels de l'estran occidental de la baie du Mont Saint-Michel. 
* Les mesures à court terme (type ALTUS) qui permettent souvent d'identifier et de discerner les forçages météo-marins et d'en quantifier les effets sur le sédiment superficiel sont directement complémentaires des mesures à moyen terme (type jalons) qui intègrent ces effets dans l'espace et le temps.

* La répartition des sédiments superficiels de même que les successions verticales de faciès enregistrées dans les carottes montrent une diminution globale d'énergie d'Est en Ouest, cohérente avec l'atténuation des courants de marée et de l'agitation, alors qu'inversement l'amplitude des remaniements est moins marquée à l'Est qu'à l'Ouest.

* A l'Ouest, les enregistrements sédimentaires (dépôt/préservation drapages vaseux) matérialisent l'influence prépondérante de la dynamique tidale de relativement faible énergie avec des courants de marée de faible amplitude et de type giratoire. Cependant l'action du vent semble importante dans les processus de remaniements des sédiments fins superficiels, gorgés d'eau. En effet, un déplacement des nappes de vases vers la baie de Cancale s'observe par vents de secteurs E - SE et inversement, les nappes se déplacent vers Hirel par vents de secteurs W- NW.

* Au centre de la Baie, à Cherrueix, les faciès majoritairement sableux sont mis en place dans des conditions de plus forte hydrodynamique liée aux courants de marée plus forts et de type alternatif, combinés à l'action des houles. En dépit de cette forte énergie, la résultante des remaniements reste faible et moins importante qu'à Cancale et/ou à Vildé-Hirel où les sédiments sont plus vaseux et donc plus facilement mobilisables.

\section{Références}

[6] Bonnot-Courtois C., Caline B., L'Homer A. \& Le Vot M. 2002. - La Baie du MontSaint-Michel et l'estuaire de la Rance. Environnements sédimentaires, aménagements et évolution récente. [The bay of Mont-Saint-Michel and the Rance estuary. Recent development and evolution of depositional environments]. - Bull. Centre Rech. Elf Explor. Prod., Mém. 26, 256 pp. TotalFinalElf - CNRS- EPHE

[3] Bonnot-Courtois C., Fournier J., Dreau A. 2004. - Recent morphodynamics of shell banks in the western part of the Bay of Mont Saint-Michel. - Géomorphologie : relief, processus, environnement, 1, 65-80.

[5] Caline B. 1982. - Le secteur occidental de la Baie du Mont Saint Michel. Morphologie, sédimentologie et cartographie de l'estran à 1/20 000. - Documents $d u$ $B R G M, 42,250 \mathrm{pp}$.

[7] Canuet F. 2003 - Analyse des faciès sédimentaires dans le secteur occidental de la baie du Mont Saint-Michel. Mémoire Maîtrise Univ. Caen, 15 p.

[4] Jestin, H., Bassoullet, P., Le Hir, P., L'Yavanc J., Degres, Y., 1998 - Development of ALTUS, a high frequency acoustic submersible recording altimeter to accurately monitor bed elevation and quantify deposition or erosion of sediments. Oceans'98IEEC/OES Conference. Nice, Conference Proceedings, vol. 1/3, 189-194.

[1] Larsonneur C. et coll. 1989. - La Baie du Mont-Saint-Michel. - Bull. Inst. Géol. Bassin Aquit., 46, 1-75

[2] L'Homer A., Courbouleix S., Beurrier, M., Bonnot-Courtois C., Caline B., Ehrhold A., Lautridou J.P., Le Rhun J., Siméon, Y., Thomas, Y. \& Villet, M. 1999. Carte géologique France (1/50 000), feuille Baie du Mont-Saint-Michel (208). Orléans : BRGM. + Notice explicative, 184 pp. 